\title{
Chapter 3 \\ The Conditions for the Development of the Properties of the Action. The Phases of the Development of the Action
}

\section{Outline of Lecture 3}

The focus of this lecture is on the measures needed to achieve the desired properties of the action. For example, consciousness of the action is achieved by the learner orienting his or her action to the identification of the important relationships between the features of the target problem. To achieve the consciousness of the action, the teacher must fully deploy the action so that the learner is able to trace what happens within each unit of the action and how the transition from one unit to the next unit occurs. Galperin argues that if a learner can trace this process, then the action acquires the meaning of a generic and not merely a subjective process. The second measure in the formation of consciousness of the action is separating the essential from the inessential aspects of the action, which can be achieved by the systematic variation of the problems offered to the learners. Finally, the third measure concerns the presentation of the action as an external process. The action is new and unknown to the learner, so it cannot be assumed that he or she will know how to perform it. Therefore, the action should be explained and demonstrated as an external objective process.

Galperin describes how other desired properties of the action can be achieved (i.e., the generalisation, rationality, criticality, and the measure of the mastery of the action). Galperin defines generalisation as identifying the key features and separating essential features from inessential. To achieve the generalisation of the action, particular attention is given to the significance of introducing a general scheme to learners, which should be actively used to solve systematically selected problems. By employing this scheme, learners identify the key features of the target concepts and the essential relationships between them. However, the main idea is that the scheme should not be memorised but understood by learners. Generalisation happens in the process of applying this scheme to a wide range of problems. The general representation of a concept cannot be conveyed to a learner; in other words, it cannot be "transferred" from one head to another. By applying a generalised scheme to different 
learning materials, the generalised meaning that corresponds to the scheme begins to stand out, and the learners start to make sense of the target concept. In the process of applying this scheme to specific problems, learners identify the key features of the concept and the relationships between them. Therefore, the process of generalisation includes two intertwined elements: the general scheme and the systematically selected material.

Three main groups of learning materials can be used to study the target concept: (i) Discipline-specific tasks; (ii) Logical tasks that differ according to their logical characteristics and contain the following: (a) all the features necessary to solve the task, (b) all necessary and some unnecessary features, (c) not all necessary features, and (d) not all necessary and some obtrusive features; (iii) Psychological tasks that differ according to the ratio of visually represented and conceptual features; that is, tasks in which the visual and conceptual features either coincide or differ. Galperin suggests offering learners different tasks based on the principles of contrast and psychological surprise to keep learners in a state of high intellectual alertness. Such principles imply the random presentation of different materials to learners.

The next property of the action — rationality/awareness—is defined as the ability to present a verbal report of the action. To achieve this property, learners complete the action through talking. As an assessment of the conformity of the defined criteria to the objective reality, criticality can be achieved by evaluating the selected criteria in terms of their compliance with reality, which then should be evaluated by these criteria. The degree of the mastery of the action can be achieved by eventually merging the small individual units of the action into bigger units and subsequently into one continuous process. Establishing the rhythm of the action is an important indicator of the mastery of the action: when the rhythm is fully established, then the speed of the action can be increased.

Finally, Galperin turns to the third subsystem: the transformation of the action into mental action. He emphasises that in mastering a new action, it assumes a higher, mental form. The transformation of the action to the mental plane of the learner happens through six consecutive phases or forms of activity:

1. The formation of the motivational basis of the action

2. The formation of the scheme of the orienting basis of the action-orientation

3. The formation of the action in its initial tangible or material form-materialised action

4. The formation of the action in loud socialised speech—communicated thinking

5. The formation of the action as silent speech-dialogical thinking

6. The formation of the action in hidden speech - acting mentally

Galperin describes the formation of the motivational basis of the action (1) as a guiding aspect of the action, which defines the significance of both (i) the action as a process and (ii) its outcome for the learner. Three types of motivation are discussed: (i) external motivation in which a learning process acts as a tool for earning a reputation of being a good learner; (ii) "sporty" or competitive motivation. By achieving desirable results, a learner develops self-confidence and is eager to achieve more and better results. A learner is eager to achieve these results; however, the process of 
learning does not interest him; (iii) inner motivation as an unselfish and insatiable passion for learning. Galperin argues that such motivation can be developed with learners. How learning emerges in each of the other phases (2-6) is presented in detail in lecture 4.

\section{Lecture 3}

In the previous lecture, we talked about the primary properties of the action, or the parameters of the action. From these primary properties we would now like to turn to the secondary properties, which are of greater interest to us. In this lecture I will describe how the secondary properties are formed from a particular combination of the primary ones.

The first of the secondary properties is consciousnessof the action. However, how do we understand consciousness of the action? As I told you earlier (it appears very clearly in experiments with animals), consciousness of the action is characterised by the subject ${ }^{1}$ orienting his action to the identification of the important relationships between the features of the problem that needs to be solved. It is important to emphasise that attention is directed to the identification of the necessary relationships between the features present in a specific task, because the features of one problem can include certain crucial relationships; while the features of another problem with the same objectives can have crucial relationships that may differ significantly.

For example, we have a problem. The features of this problem are related in some way to each other, and some of these relationships are crucial for solving this problem. We have to identify these crucial relationships and present them as a basis for the orientation of the actor. We can do so by applying a combination of the following measures. First, we have to deploy the action fully so that all its constituent individual units are completed. We cannot skip a single unit, as a learner will not be able to compensate for this unit himself and this will naturally create a gap in the sequence of the units. If this were to happen the action would not present itself as something performed consciously because the learner would not be able to understand why, after a gap, there follows another unit of the action. So, the first measure is to fully deploy the action. The term "a fully deployed action" is relative, because what is the sufficient deployment of an action will vary for different learners. Therefore, we should identify the required degree of deployment of a new action for a particular group of learners. To deploy an action means that the learner should be able to trace what happens within each unit, and how the transition from one unit to the next one occurs. If a learner is able to trace this process, then the action acquires the meaning of an objectively necessary process.

\footnotetext{
${ }^{1} \mathrm{~A}$ subject can be also understood as a learner. Subject (noun) is masculine in Russian.
} 
However, that is not all. Any material ${ }^{2}$ has many aspects that may be irrelevant for a particular action. Therefore, the second measure for the formation of the consciousness of the action is the purification of the action from minor and insignificant aspects. In our conventional language, this is called differentiation, separating the essential from the inessential. How this is achieved, I will explain in detail later, but for now I shall mention that this can be done by a systemic variation in the problems offered to learners that can be solved by performing a particular action.

The speed at which a learner is able to trace the sequence of the transformations that happen to the initial material during this action is extremely important. Finally, the third measure that is always assumed, but which needs to be made explicit, is that the action in its successive units should be presented as an external objective process, not on the mental plane. We are dealing with a new action unknown to the learner, and we need to present this action as fully deployed as possible. This is absolutely necessary because the units of the action and their corresponding transitions are unknown to the learner. We cannot simply assume that the learner will get an idea of how to perform this action himself; we have to unravel the action and demonstrate it as an external objective process.

Now you see how many measures we have to pursue in order to help form the consciousness of the action with learners. This means that we have to create such an image of an action that would empower a learner to perform the action according to its key features and their objective relationships.

The second property is a generalised action. Generalisation can be understood in different ways. In our case, we are referring to generalisation in the sense of identifying the key features and separating the necessary relationships between these features from those that are inessential. As you will remember, this also constitutes the consciousness of the action and is achieved in two ways.

We used to believe that a new action, or more generally a new phenomenon, which is introduced to the learner, has to be demonstrated by a clear and simple example. It turns out, that this is not the best way to go about it, because a concrete example, simple and clear for the teacher may not be so simple and clear for the learner. The significant relationships are connected with the specific content of a particular example. Even if the example is simple (for the teacher), it is nevertheless associated with the specific content. Practice shows (I will tell you more about this later) that no matter how much you emphasise that this is a just an example and that the learners have to identify some general relationships, still this particular example gets unconsciously fixed and is remembered by students. Therefore, later on we have to get rid of the specific content of the example that we used in our explanation.

A better way is not to use a specific example (even if the example is very simple), but instead offer a (general) scheme. This scheme can be applied to all the cases where a particular rule can be used, or in all the cases of the target phenomenon. In this sense, the scheme is general; however in itself it is a specific schematic representation of some relationships. The main idea is that this schematic representation should not be simply memorised but should be understood by learners. I have already

\footnotetext{
${ }^{2}$ Material in the meaning "learning material".
} 
mentioned that a traditional approach to teaching and learning with its reliance on prior memorising of knowledge for its subsequent application is very unfortunate. A better way is creating a scheme (which can be, for instance, in a form of a table) and then applying this scheme for solving systematically selected problems. Generalisation happens, in fact, in the process of application of such a scheme to a wide range of problems.

The view that emerged from our assumptions, that it is possible to give a (general) representation of a concept at the beginning of learning is, in fact, wrong. This (general) representation cannot be supplied to a learner. It cannot be "transferred" from one head to another, because initially you can show, at best, an abstract scheme without any specific content. Although originally this scheme was something quite specific and concrete, it appears to the learner as a schematic drawing, and nothing else. Only when you apply this scheme to varied material, does the generalised meaning that corresponds with the scheme begin to stand out from the material, and only then do learners start to make sense of this (general) representation. In the process of the application of this scheme to specific problems the learners identify the key features and the essential relationships between them.

Therefore, the process of generalisation comprises two intertwined elements: the first is the (general) scheme, and the second is systematically selected material.

However, how do we select this material? There are three main groups of learning material that are used for studying a specific rule or a concept.

The first group is specific for each subject. These are discipline specific types of material. I remember one of the studies: it was about teaching children to find the subject in sentences. It is important that the subject in the Russian language can be expressed with different parts of speech, including a verb in its infinitive form. Of course, these differences create certain difficulties which must be overcome.

So, the first group - are the discipline specific types of material. I would like to emphasise that these are different types, not just variations within one type, because, for example, a big book of problems, with many different tasks, in fact, contains only very few types of tasks. Therefore, there are types of tasks, and there are different tasks that belong to the same type. For example, a numeral can be a subject in a sentence, as in the saying: "Seven do not wait for one." Here "seven"-is a subject. Well, you may also say: "Five do not wait for one" This is a variation: seven-is not five, but this variation is of the same type. You can create many other similar variations. However, we should remember that only a difference in types has its differentiating value. So, the first group is different types of discipline specific material.

The second group are the materials that are different according to their logical characteristics. Each concept, including the concept of an action is characterised by a set of its essential features. ${ }^{3}$ You may have material, ${ }^{4}$ which contains only the necessary features or you may have material which contains all those that are necessary and, in addition, some unnecessary features. This is important because you need to identify not only all the present features, but the features that are crucial

\footnotetext{
${ }^{3}$ In a mathematical problem, the features are the conditions of the problem.

${ }^{4}$ Learning material, e.g. in the form of tasks.
} 
for this action. Another possibility may be when not all the necessary features are to be found in the material. Strictly speaking, this means that the concept cannot be recognised because some of the features are missing; consequently, the target phenomenon fails to address the concept. Finally, the fourth type of material is when not all the necessary features are to be found in the problem and there are many unnecessary, obtrusive features. This means the learners are not supplied with all the necessary features, and they are also given many unnecessary and obtrusive features and they are required to make sense of all this information.

In sum, we have four logical types that contain:

1. All necessary features;

2. All necessary and some unnecessary features;

3. Not all necessary features;

4. Not all necessary and some obtrusive features.

A great variety of specific material should be selected for each of these four logical types.

Finally, the third group are psychological types of material. Psychological types differ according to the ratio of visually represented and conceptual features of the material. On the one hand, a task can contain a combination of these two, when visual features and conceptual features coincide (the easiest and the most enjoyable case). For example, there are tasks in which visual and conceptual features differ. You may have a situation where a feature is present in the visual representation, but conceptually it is not there. For example, when we were teaching children the concept of a perpendicular, the learners were given a picture and were told that the marked angle was $90^{\circ}$ (Fig. 3.1).

The difference was only in one degree that could not be noticed with a naked eye and it seemed that the angle was right, but in fact, it was not right, but sharp. There was a discrepancy between the visual and conceptual features.

Fig. 3.1 The learners were informed that the marked angle was $90^{\circ}$

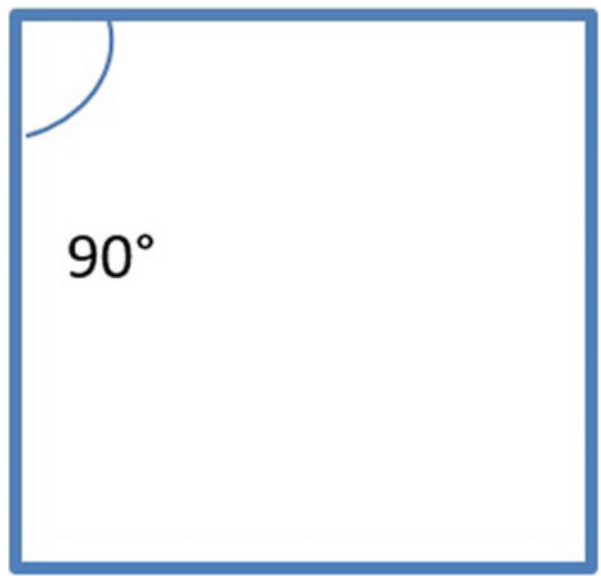


Fig. 3.2 A figure of a "circle"

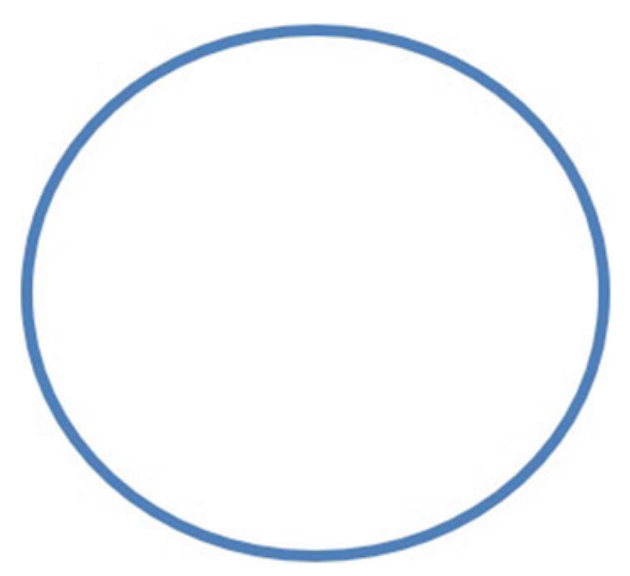

We may also have the opposite situation. Let us say we draw a figure and say that it is a circle (Fig. 3.2).

We can see that this is not a circle, but the task says that this is a circle. Correspondingly, in a circle all the radii are equal. Working with the figure learners have to come to an understanding that if they were told, that this was a circle, then all its radii were equal.

In sum, you have to select three types of material that belong to a psychological type:

1. When the visual and conceptual features coincide;

2. When the visual and conceptual features diverge: in one case you have the presence of these features visually; however, conceptually these features are absent.

3. The opposite case: the features are not present visually, but you are told that you are given, for example, a circle with all the range of its properties.

To sum up, there are three big groups of material that should be selected and offered to learners: (i) Discipline specific; (ii) Logical; and (iii) Psychological.

Each of these groups requires a selection of particular material in a sufficient amount: we need to fill each of these groups with its own specific content.

Since we have such a large number of different groups or types of material, traditionally there arises a problem of organising this material in terms of the order in which these groups or types should be introduced to learners. A common approach in traditional teaching and learning is to go from simple to difficult, but, as we know, traditional education does not provide a learner with reliable tools for solving problems. Traditional teaching relies heavily on the learner's individual guess at what to do and how to approach learning. Well, the result of such an approach is well known. In our approach, we provide a learner with a reliable tool-the scheme of the orienting basis of the action and different groups or types of materials. Since we provide this reliable tool, then the requirement of a gradual movement from simple to complicated becomes redundant because all the tasks can be solved with the help 
of the complete scheme of the orienting basis of an action. In this case, we are facing different requirements: not to move from simple to complicated, but another psychological approach previously identified by Pavlov should be followed. This is an approach based on the principle of contrast, psychological surprise to keep the learner in a state of high intellectual liveliness and intellectually alert.

To begin with, you offer a learner a problem at a low level of difficulty; you may even offer a simple task, which is suddenly followed by one of the most difficult tasks so that the learner does not relax and start thinking that everything can be solved so easily. Then offer a task, for example, with an incomplete set of features so that the learner cannot think that everything is ready for him and he needs only to perform the action. He needs to evaluate whether all necessary features are there. If not all the features are present in the task, a learner has to say that the problem cannot be solved, because such-and-such features are not found.

Therefore, the main principle of the sequence of problems offered to learners is the random presentation of different types of material and maintaining of a high level of tension in the orienting activity of the learner. Since we do not use prememorising and recognise that learning happens in the course of the action, in the process of solving problems, we need to have a great number of various problems in our repertoire.

Of course, you cannot rely on yourself being able to figure out quickly everything you need during teaching. It is necessary to arrange the order of the material in advance. We do it this way: we simply enumerate the tasks in the order they will be offered to the students. First, we select a large number of tasks. It is much better to have "leftovers" afterwards then to find ourselves in a situation when we do not have enough tasks. We write numbers on these tasks in advance in the order we will offer them to the students, so that there is no need to worry about that later. Then the tasks are presented according to the assigned numbers. The order of the tasks has to be thought through carefully and checked in advance whether it works well.

If we combine two conditions: give the complete orienting scheme of the action and immediately proceed to solving of the selected problems using this scheme, in doing so, we ensure the desired degree of generalisation. This implies that we cannot say, as it usually happens in traditional education, that a student is capable of doing something, but to a very limited extent. This just cannot happen and if this happens, it is our fault because we have not provided something for the learner.

We identify the levels of generalisation to be applied to the rule, and select the problems that would be directed towards the formation of this level of generalisation with learners. In this case, a student cannot help generalising. We force him to complete this action in its fullest, and the learning outcomes do not come as a surprise. These outcomes have been anticipated.

Now the next property is rationality, awareness. These are terms that have an infinite number of interpretations. That is why we are not going to deal with all these interpretations, but simply agree on a particular interpretation of this notion, which is a right of any researcher. The only thing is that the researcher has to let us know which interpretation: "I understand this notion in such—and—such way". You can 
disagree with me and say that perhaps another word should be used, but if I explain, what I mean by rationality, none can contradict me.

We understand rationality/awareness as an ability to present a verbal report of the action. The question now is how to ensure this property. It is very important that at the beginning of the action, a person explains what he is doing and why he is doing it. We will listen to what he is saying, and correct him if he is saying something wrong, even in the case of him performing everything correctly. This actually happens very often and we cannot allow such inconsistency. What is important is that students should not learn the most economic and perfect wording by memorising ready-made phrases and formulations. This should not happen, because a speech stereotype can be formed without any relation to what a learner is actually doing. Speech should be an active reflection of an objective process. In order to teach this active reflection, a learner should use a large variety of his own words and even when speaking with his own words, but rather monotonously, he should be told-“You know, dear, I have already heard you speaking that way. Try to speak, with more engagement, not so monotonously, not so boringly". It is curious to note that this process resembles other more subtle processes in other situations.

Apparently, Stanislavsky when rehearsing a new play, demanded that the actors read the play and, in particular, their parts in advance. At the rehearsal, Stanislavsky would not allow the actors to speak with the words written in the script. The actors had to play their parts using their own words. Only after they had played a certain part using their own words, he gradually transferred them to the words of the author of the play. Otherwise, the fixed words of the play would have turned into a speech stereotype that you can learn to enunciate, without thinking. This would have resulted in the actor mouthing some words but acting something different. It is necessary that speech itself actively reflects the situation. When this has been achieved, only then one can use other words. At this point you come to the specific speech formula and this process should accompany material action.

You see, at first, it is necessary to speak with your own wide vocabulary and when the speech formula has been "played around with", you can move on to the standard definition. In this way, we ensure the rationality of the action.

Now criticality. This is a difficult property. I have already told you that criticality is the assessment of the conformity of the defined criteria to the objective reality. Very often, we do not notice that certain features predefine some of the criteria that they are based on, and if we doubt these criteria then these features will be invalid. Recently I discussed a published book with its author, who believes that not everything is ideal in the human mind, that some part of mental activity should not be called ideal. I asked him: "Excuse me, but what are your criteria of ideal? What do you call ideal?" He said that ideal is, in fact, everything that comes from ideas. Well, since animals do not have any ideas, this means that the entire mind of an animal is not ideal. I told him: "Well, in this sense, it is banal, isn't it? We have known for a long time that animals do not have concepts. Does this mean that an animal's mind is something which is not ideal? Ideal should be understood in another sense".

This means that a person takes a criterion that he does not have a name for, that is only implicit, and then on the basis of this criterion he presents a risky statement 
such as mind is not ideal. I asked him: "Well, what is it then? Material?" To which he replied: "No, not material, but it is of itself". It is neither ideal, nor material, but something of a third nature. The main point, as it turned out, is that he had taken the concept of an idea in a human sense as the criterion of ideal. This is a narrow and simply a wrong criterion.

It is important to notice that very often we do not analyse the criteria we proceed from. They appear to us as something natural. This is always the case when we take the perspective of a criterion; you see things from this perspective. Therefore, understanding criteria is very important as in many cases criteria are adopted intuitively. Above all, the criteria should be evaluated in terms of their compliance with reality, which, in turn, should be evaluated by these criteria. Thus, ensuring criticality is a very difficult and demanding task, but it should be done by selecting the criteria, which are often being used intuitively and unconsciously, recognising that sometimes such an approach to selecting criteria is unjustified.

The next property is the degree of mastery. This is a particularly important property and I am going to explain why. After all, we believe (I will talk about it later) that the mastering of the action is not just memorising it in the form in which it is being explained and performed. There is a continuous change in the form of the action itself. You cannot move to the next higher form, until you recognise that the previous one has not been developed sufficiently. However, we cannot linger on these transitional forms for too long either, because every development means mastering the action in this particular form, which is only transitional, not yet the final one.

We conducted a study with children at the end of their first year of school and explored the difference between the underachieving students (those who usually get a grade $t w 0^{5}$ ) and those who are usually awarded a three; the difference between the grade three students and the grade four students, etc. We found out that underachieving students differ from achieving pupils not in the way they think. Underachieving students do not think poorly, sometimes they think very well, but they do their thinking in early and highly uneconomical forms.

Imagine that a child has a delay in counting, which means that he can only count objects and things. He uses his fingers for counting, which is extremely convenient, as with Russian counters that have tens, and the child can clearly distinguish fives on both hands. Therefore, fingers can be used for counting and this child is holding his hands somewhere under the desk and is counting with an incredible speed, bending his fingers. We were holding his hand and still were feeling the movement of his fingers, but we could not distinguish anything because he was moving them so fast when counting. His counting was absolutely correct; he did not make a single mistake. However, if we squeezed his hand so hard that it was difficult to move his fingers, he stopped counting with his fingers, and began to count using other methods: he counted spots on the blackboard, on the desk, on the walls, etc. He could perform any calculations, but only with objects. When he was given a task with numbers greater than ten, he would count his fingers several times and give the correct answer, but the teacher said, "He thinks so poorly and slowly". In fact, he thinks quickly, he thinks

\footnotetext{
${ }^{5}$ On the scale one (poorest)-five (best)—-the grading system in the USSR.
} 
with a speed of light, but just counted slowly because he was using his fingers. This is the delay in the earliest and first necessary forms of the action. If he remains in these forms for too long, they become the reason for his delay. The trouble is that this gap is not immediately clear to the teacher. Poor little boy, he is struggling to keep up with the others. How can he possibly do it? Only by automating his way of counting as much as possible. He reaches almost perfect virtuosity in performing these activities, but in doing so, he gets more and more "stuck" in this hopeless way of solving problems.

There is a danger of the opposite: intermediate forms of action should not be completely mastered. They need to be brought to a certain degree of mastery no more and no less. Otherwise you will not be able to transfer to the next forms of action. What indicates the mastery of the intermediate forms of action? Initially, an action appears to the child as a series of separate, independent links which are performed in such a way that the child always first inspects the closest link, which he has to perform. Once this link has been inspected-the child begins to complete it, and then he proceeds to the next link, stops again, performs an orientation to the next part of his work, and then completes it too.

In such a way an action is first being divided into independent links, separated by the periods of orientation. Then these links begin to merge into bigger units: instead of two small links, there appears one unit. Hence, the formation of bigger units happens. The mechanism of how smaller links merge-is a very important aspect of theoretical significance. I will tell you more about it later. In the meantime, it is important for us to understand that the original small links merge into bigger units or steps. This does not happen immediately all the way through, but, naturally, first in the easiest links. Later these bigger units also merge, until the action starts to flow as a continuous process without any pauses at individual units.

This is the first phase of mastering the action. Then the following happens: before this phase, the action cannot be performed either with the required rhythm or at the required speed. When, for the first time, the action begins to flow as a continuous process, then its rhythm can be identified, however it is still slower than the final version of it.

Establishing the rhythm of the action is a very important indicator of the mastery of the action. When the rhythm is fully established, then the speed of the action can be increased.

All these parameters: integrity, continuity of the action, its speed and rhythm—are the indicators of the mastery of a new action.

When we are at an intermediate stage, this means that we have not achieved the complete mastery of action. However, it also means the mastery of action in this particular form. We need to move on, because once the action gets its continuous flow, we try to transfer it to the next higher level. Maximum automation is achieved only when the action reaches its final shape. Without such automation, excellence in the performance of the action cannot be achieved. I will tell you about the nature of psychological and physiological mechanisms of automation when we talk about the most representative actions for this case - physical actions. 
So far, we have looked at two large subsystems - the subsystem of the conditions for the formation of a new action, and the subsystem of the conditions for the acquisition of this action, the desired properties. Now we have come to the third and the final subsystem of conditions - the subsystem of the transformation of the action into mental action. Actually, this subsystem has given its name to the whole process- the phases of the development of mental actions, although it certainly does not represent the whole structure of the system.

To master a new mental action, means to ensure the formation of this action in its highest, mental form. In order to do so, we need to trace the path of its development from the beginning. We can do it through six consecutive phases. I will list them first, and then we will focus on what happens at each phase and what we obtain in the end.

The first phase - the formation of the motivational basis of the action.

The second phase-the formation of the scheme of the orienting basis of the action.

The third phase - the formation of the action in its initial material or materialised form.

The fourth phase - the formation of the action in externalised social speech.

The fifth phase-the formation of the action in outer speech "with yourself".

The sixth phase - the formation of the action in hidden speech.

Let us have a look at what happens at each of these phases.

The first phase is very discreet and, I would say, even neglected. I should confess that I and my colleagues have been neglecting this phase for a long time, believing that no matter how and by what means we motivate the learner, the main idea is to make him do what we would like him to do. The type of motivation does not really matter. For example, whether the child is working for a chocolate, or for the sake of being praised, or simply because of the fear of the adults - this does not matter. We used to believe that the type of motivation did not matter; however, we were wrong. The effect of a particular type of motivation is a delicate thing, which is not easily detected, but in the end it can have severe consequences.

Motivation is often viewed simply as a power unit, as something that drives, propels the work of the learner. To be honest, we used to think of it in that way for a long time. Even such a talented modern foreign psychologist as Jean Piaget, has the same understanding of motivation he believes that motivation is the source of energy that drives the behaviour. Hence, a motive-so to say, is the reason, the justification of the amount of effort a learner puts into his work. However, this turned out to be wrong. Motivation is not only the energy, although the energy of the learner is what we can actually observe. Motivation is an orienting aspect, a guiding aspect, identifying in the target object of the action and in the action itself what is important for the learner. Motivation determines the value of the tools used to perform this action, and the stability of this action, and much, much more.

Recently we had a case. We taught children with lack of attention how to improve their attention. In general, this lack of attention was eliminated in almost all the children we were teaching, but there were two boys we did not succeed with. Only two boys! These boys, however, worked with the experimenter very well, like the 
other children, following all the instructions. Only later we found out why they could not master the action that would improve their attention. Apparently, the father of one of the boys persuaded his son that no matter whether you are a scientist or not, no one can learn without making mistakes. Well, if no one can learn without mistakes, what is the point of trying not to make them? If one is doomed to be making mistakes when learning, there is nothing to worry about. However, with the experimenter the boy worked well doing everything correctly. Why was it so? Because, as it turned out, he was very pleased that out of the entire class it was him who was invited to work individually with an adult. They were just children of the 2nd-3rd grades: the boy was flattered that no one else was invited, but he was. He enjoyed working with the experimenter. Nothing, unfortunately, arose from this feeling, as the boy did not see the point in mastering the activity; he just did not need to. This is a type of motivation: he works well for the experimenter, but he does not need it himself, so mastering the action does not happen.

And here's another boy we did not succeed with. Though he used to make mistakes, he used to correct them, getting no more than grade "four". He believed that grade "four" was a good enough grade for him and so why would he try and get a "five"? The boy did not have the motivation for learning how to perform better. With the experimenter he worked like everyone else, properly, but as soon as he was out of the direct interaction with the experimenter, he began to work as he had done previously.

The most important thing is that we may not take into account motivation, but it is still there and determines the degree of success of the educational process.

We have previously identified three types of motivation. The first type is that the learner is studying to achieve something that comes from the outside: for example, that his mother would give him the money she promised for the cinema, or he knows that he will be praised for good effort, etc. The child does not mind learning, but he does not like it himself. The important thing is that there is an external and more interesting life, and the true motives of his learning are in this external life. The learning process with this type of motivation acts as a tool for earning his reputation of being a good learner. This is an external type of motivation.

There is another type of motivation which is more interesting. It happens when you give the learner all the tools for solving problems and then, moving from task to task, the learner grows in confidence in himself and his abilities. Gradually the learner becomes competitive with his friends and even with himself. He develops the type of motivation, which may not be entirely accurately labelled as a "sporty" or competitive motivation. The aim is the achievement of the desirable result and the learner develops self-confidence and the respect of his peers, because he keeps achieving better and bigger results. The learner is eager to achieve these good results, but in the process of learning, knowledge does not interest him, only the results. This is a so-called "sporty" interest or motivation.

Finally, there is the last type of motivation which the most difficult to achieve, but it is truly valuable. This is some special unselfish and insatiable passion for learning. Very often they say that there are some strange people who do not have any ordinary demands in life, but a great love for learning. When I was a little boy, I used to know an elderly man who loved the process of learning so much that he used to say: 
"When I die, please write on my grave: his soul craved for knowledge". In fact, it is wonderful to enable a student to be this strange person even for a short time, because no other motivation can replace this urge for learning. The most interesting thing is that people are not born with this urge, but it can be developed like other things. I will tell you how to form this type of motivation at the very end of this course. In the meantime, you have to believe me that this is possible. This inner motivation (as we label it) for learning cannot be replaced by anything else, because we certainly cannot stand by the person and repeat: "Read, read, read " if he does not really feel like reading.

In sum, today we distinguish three types of motivation, and it is important that these types can be formed and taught, although not always deliberately.

Open Access This chapter is licensed under the terms of the Creative Commons Attribution 4.0 International License (http://creativecommons.org/licenses/by/4.0/), which permits use, sharing, adaptation, distribution and reproduction in any medium or format, as long as you give appropriate credit to the original author(s) and the source, provide a link to the Creative Commons license and indicate if changes were made.

The images or other third party material in this chapter are included in the chapter's Creative Commons license, unless indicated otherwise in a credit line to the material. If material is not included in the chapter's Creative Commons license and your intended use is not permitted by statutory regulation or exceeds the permitted use, you will need to obtain permission directly from the copyright holder. 